EUROPEAN ORGANIZATION FOR NUCLEAR RESEARCH

European Laboratory for Particle Physics

Large Hadron Collider Project

LHC Project Report 515

\title{
THE TEST FACILITY FOR THE SHORT PROTOTYPES OF THE LHC SUPERCONDUCTING MAGNETS
}

\author{
W. Venturini Delsolaro, A. Arn, L. Bottura, C. Giloux, R. Mompo, A. Siemko and L. Walckiers
}

\begin{abstract}
The LHC development program relies on cryogenic tests of prototype and model magnets. This vigorous program is pursued in a dedicated test facility based on several vertical cryostats working at superfluid helium temperatures. The performance of the facility is detailed. Goals and test equipment for currently performed studies are reviewed: quench analysis and magnet protection studies, measurement of the field quality, test of ancillary electrical equipment like diodes and busbars. The paper covers the equipment available for tests of prototypes and some special series of LHC magnets to come.
\end{abstract}

LHC Division

Presented at the 2001 Cryogenic Engineering Conference and International Cryogenic Materials Conference CEC/ICMC 2001

16-20 July 2001, Madison, Wisconsin, USA

Administrative Secretariat

LHC Division

CERN

CH - 1211 Geneva 23

Switzerland

Geneva, 19 October 2001 


\title{
THE TEST FACILITY FOR THE SHORT PROTOTYPES OF THE LHC SUPERCONDUCTING MAGNETS
}

\author{
W. Venturini Delsolaro, A. Arn, L. Bottura, C. Giloux, \\ R. Mompo, A. Siemko, and L. Walckiers \\ CERN, CH-1211 Geneva 23, Switzerland
}

\begin{abstract}
The LHC development program relies on cryogenic tests of prototype and model magnets. This vigorous program is pursued in a dedicated test facility based on several vertical cryostats working at superfluid helium temperatures. The performance of the facility is detailed. Goals and test equipment for currently performed studies are reviewed: quench analysis and magnet protection studies, measurement of the field quality, test of ancillary electrical equipment like diodes and busbars. The paper covers the equipment available for tests of prototypes and some special series of LHC magnets to come.
\end{abstract}

\section{INTRODUCTION}

The Large Hadron Collider (LHC), the next accelerator being constructed on the CERN site, is based on superconducting twin aperture magnets operating at $1.9 \mathrm{~K}$. The development program for the LHC main dipole magnets started in 1988, based on single and twin aperture $1.3 \mathrm{~m}$ long models [1]. More recently, several types of strong focusing quadrupole magnets were developed for the dispersion suppressor and interaction regions of the accelerator. A cryogenic test facility ("Block 4") was assembled initially to test the short dipole models [2]. To cover the increasing test needs the facility has been constantly upgraded [3], [4]. In 1992 a second vertical cryostat was installed, followed by a third one in 1995. The paper is focused on the description of the "Block 4" test facility, its performances and potentialities. The layout, operation and capabilities of the instrumentation tools are analyzed in sections 2-5. Recent and planned modifications needed for the LHC baseline magnets are presented and conclusions are drawn.

\section{LAYOUT OF THE INSTALLATIONS}

The cryogenic architecture of the installation is composed of a central storage dewar of 20001 capacity for liquid helium at $4.2 \mathrm{~K}$, connected to the cryostats via a central distribution box (FIG 1). Each cryostat has three main cryogenic ports: a $4.2 \mathrm{~K}$ filling line, 


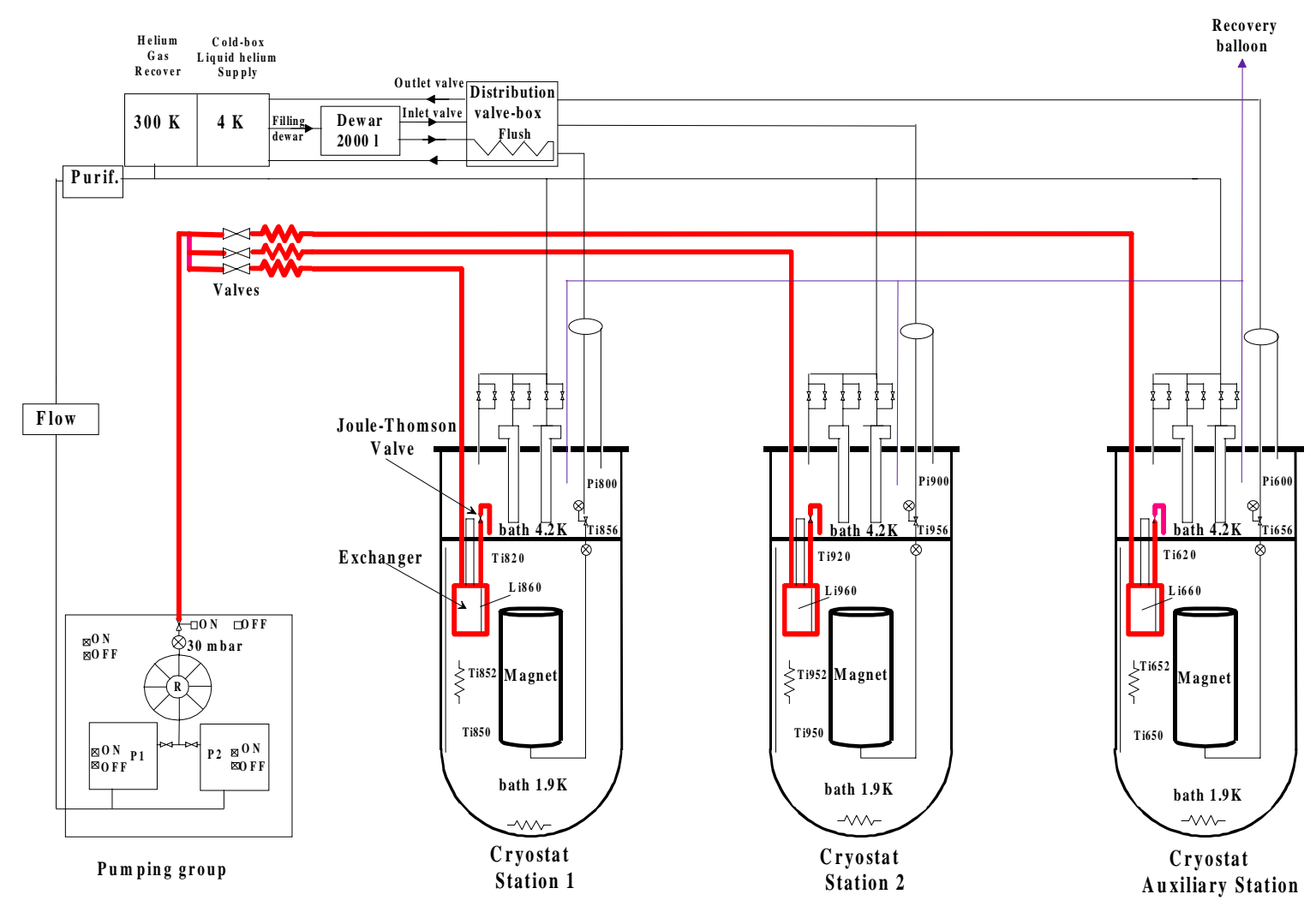

FIGURE 1. Layout of the test facility (operation at $1.9 \mathrm{~K}$ ).

a pumping line and a recovery line. The pumping speed of the Roots group is $1.6 \mathrm{~g} / \mathrm{s}$ at 24 mbar. After online purification [5], the pumping line is connected to the recovery line back to the helium liquefier, which, in turn, feeds the central dewar. An $80 \mathrm{~m}^{3}$ capacity balloon is predisposed to receive excess helium evaporated during transients to normal state (quenches) of the superconducting magnets under test. The test facility comprises 4 vertical cryostats, 3 of which operate at superfluid helium temperature. Some technical parameters of the main cryostats are given in TABLE 1. The fourth cryostat is not connected to the pumping group, and allows only operation at $4.2 \mathrm{~K}$ and 1 bar. It is used for the cold tests of LHC by-pass diodes [6]. The helium circuit is integrated into the cryogenic network of the CERN north area.

TABLE 1. Technical parameters of the three main cryostats

\begin{tabular}{lrrrr}
\hline \multicolumn{1}{c}{ Cryostat } & & Station 1 & Station 2 & Station 3 \\
\hline Useful height & {$[\mathrm{mm}]$} & 1498 & 1865 & 2300 \\
Useful diameter & {$[\mathrm{mm}]$} & 800 & 850 & 300 \\
Current leads & $(18 \mathrm{kA})$ & 2 & 2 & - \\
Current leads & $(2 \mathrm{kA})$ & - & 2 & 4 \\
Current leads & $(800 \mathrm{~A})$ & - & 2 & - \\
Current leads & $(100 \mathrm{~A})$ & - & 8 & - \\
Cool-down time & $300 \mathrm{~K}-4 \mathrm{~K}$ (for a short dipole) & $42.5 \mathrm{hrs}$ & $13.5 \mathrm{hrs}$ \\
Cool-down time & $4 \mathrm{~K}-1.9 \mathrm{~K}$ (for a short dipole) & $5.5 \mathrm{hrs}$ & $12 \mathrm{hrs}$ & $2.5 \mathrm{hrs}$ \\
\hline
\end{tabular}




\section{COOL-DOWN AND OPERATION}

The three main cryostats work according to the principle of Claudet [7], consisting of two liquid helium baths at atmospheric pressure separated by a lambda plate. Magnets under test are immersed in the lower bath at the operation temperature of $1.9 \mathrm{~K}$. The main heat load is intercepted at $4.2 \mathrm{~K}$ in the upper bath. After direct transfer from the storage dewar to the lower bath, pumping on the lower bath heat exchanger (LBHE, see FIG 2) initiates the cool down to $1.9 \mathrm{~K}$. The liquid coming from the upper bath is sub-cooled in a first counter-flow heat exchanger down to $2.17 \mathrm{~K}$ at the inlet of the Joule-Thompson valve, where it is flash evaporated and expanded in the LBHE. Heat is removed from the lower bath through the heat exchanger's wall. Cool down to $1.9 \mathrm{~K}$ is accomplished in three stages, during which three different control loops are active (cf. FIG 3 and FIG 4). The control acts by throttling the Joule-Thomson valve. In the first stage, when all liquid entering the LBHE is vaporized and its temperature is much lower than that of the bath, the control variable is the total helium flow. Subsequently, liquid is formed in the LBHE, and temperatures tend to equalize: the control switches to liquid level, and pressure is lowered to reestablish a temperature gradient between the LBHE and the lower bath. The third stage sets in when the cool down to $1.9 \mathrm{~K}$ is almost achieved. During steady operation the system manages small temperature fluctuations by directly controlling the lower bath temperature.

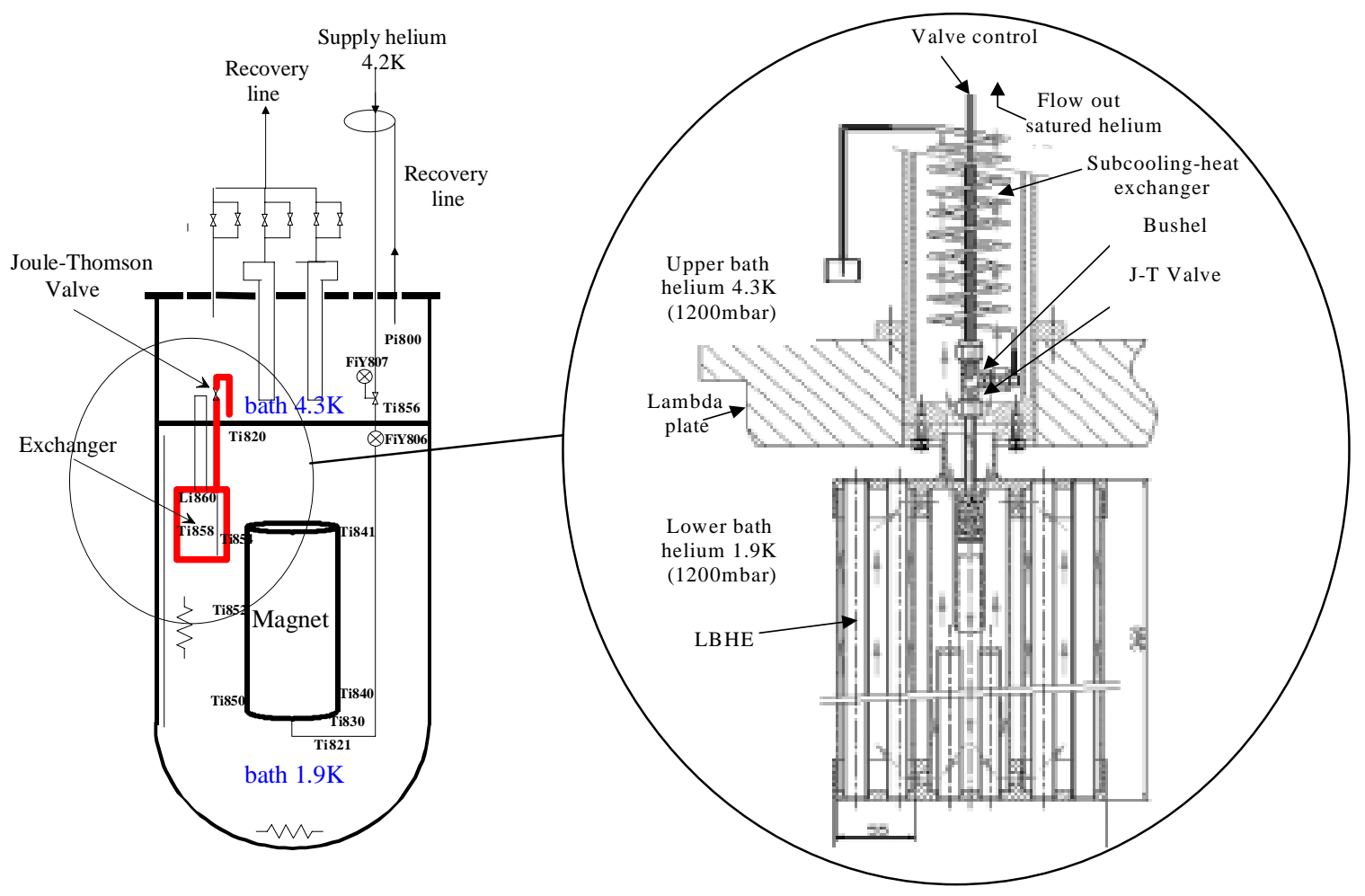

FIGURE 2. Claudet bath: detail of the sub-cooling and lower bath heat exchangers. 


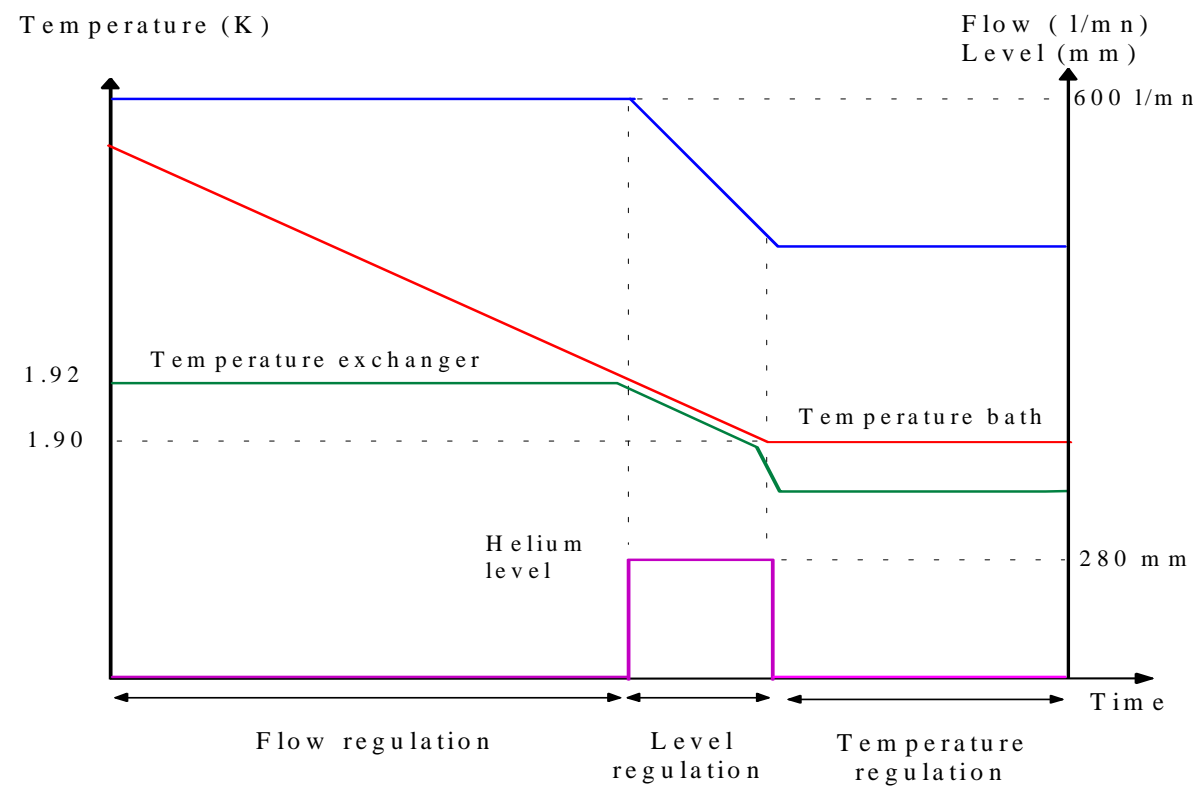

FIGURE 3. Schematic phases of cool down to $1.9 \mathrm{~K}$.

The whole sequence is programmed in a standard PLC (Siemens, S5-95U). The regulation of flow is active as long as the temperature is higher than a set point and the liquid level in the LBHE is below a threshold. When this threshold is overcome, the regulation of level operates until the set point temperature is reached. The resulting cool down time is optimized for a given maximum total flow [8]. Sub-cooling to $1.9 \mathrm{~K}$ of one cryostat is possible during operation at $1.9 \mathrm{~K}$ of a second cryostat.

\section{Station 1}

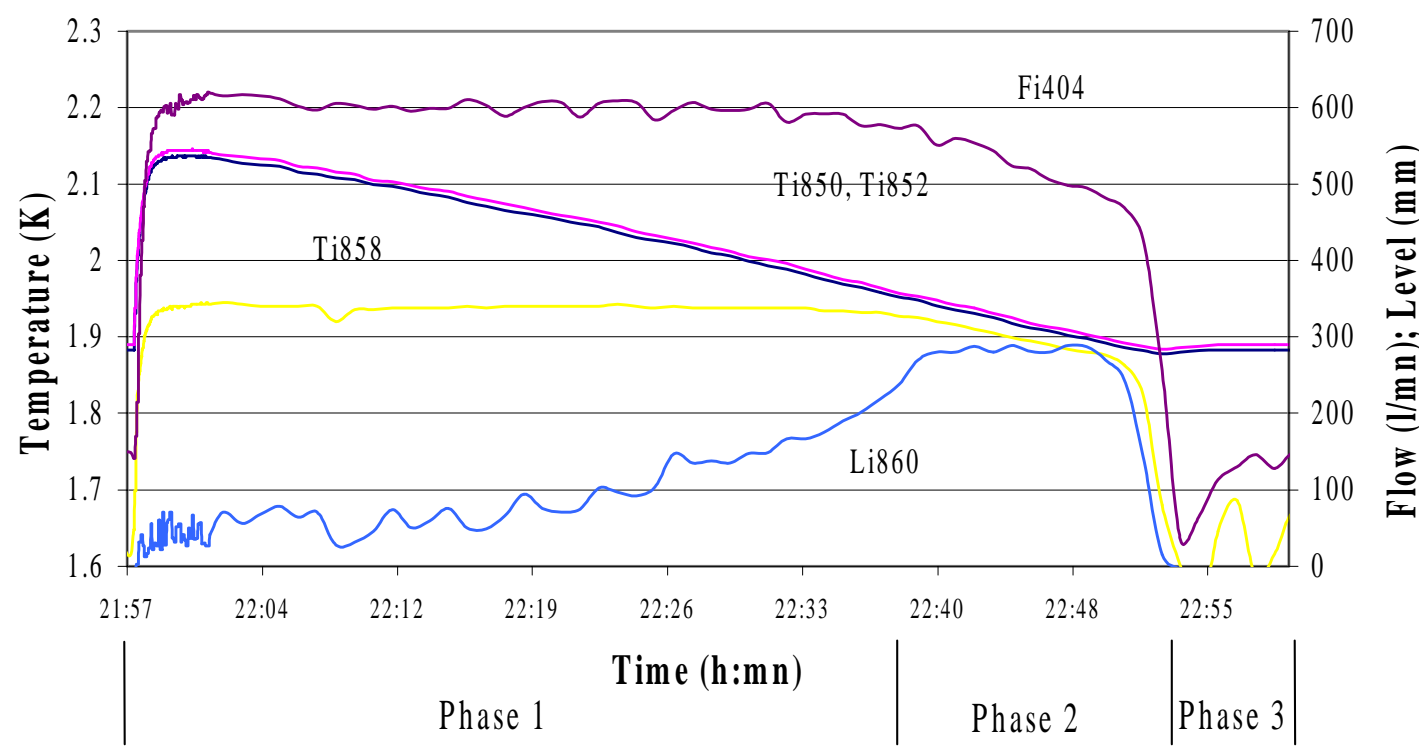

FIGURE 4. Cool down of Station 1 after a quench. Fi404 helium flow; Ti850, Ti852: lower bath temperatures; Ti858: temperature in the lower heat exchanger; Li860: helium level in the heat exchanger. 


\section{POWERING, QUENCH AND MAGNET PROTECTION TESTS}

Superconducting magnets are complex systems whose main properties are dependent on thermal and powering history. As a consequence of magnet training phenomena [9], the evaluation of power performance requires a full series of quenches to be carried out in controlled conditions. Nominal currents of magnets tested in the facility are ranging from few tens of A for some of the LHC corrector magnets up to $11800 \mathrm{~A}$ for LHC dipole models. Short model dipoles were regularly trained up to $14000 \mathrm{~A}$, and a $\mathrm{Nb}_{3} \mathrm{Sn}$ dipole model required powering up to $19800 \mathrm{~A}$ to reach the field of $11.3 \mathrm{~T}$ [10]. So-called natural (or training) quenches are performed by raising the magnet current with a low ramp rate $(10 \mathrm{~A} / \mathrm{s})$, until onset of the transition to normal state. Several power supplies adapted to different inductive loads are available in the test Station (TABLE 2). Accuracy of current in the ppm range and very low ripple noise on current plateaus are required for magnetic measurements.

TABLE 2. Main power supplies available in the test station

\begin{tabular}{ccccc}
\hline Number & Maximum current & Maximum voltage & Accuracy & Ripple output voltage \\
\hline 1 & $20 \mathrm{kA}$ & $20 \mathrm{~V}$ & $10 \mathrm{ppm}$ & $200 \mathrm{mV} \mathrm{pp}$ \\
1 & $\pm 2 \mathrm{kA}$ & $\pm 10 \mathrm{~V}$ & $10 \mathrm{ppm}$ & $150 \mathrm{mV} \mathrm{pp}$ \\
2 & $\pm 600 \mathrm{~A}$ & $\pm 12 \mathrm{~V}$ & $10 \mathrm{ppm}$ & $4 \mathrm{mV} \mathrm{pp}$ \\
\hline
\end{tabular}

The quench acquisition system is based on a commercial multi-channel transient recorder (Nicolet Technologies ${ }^{\circledR}$ ) and related software running on PC. It can handle up to 300 synchronized channels, with sampling frequency from $160 \mathrm{~Hz}$ up to $300 \mathrm{kHz}$. Analog signals from the test set are amplified and sent to the recorder, where they are digitized and memorized in a ring buffer. A set of few key channels is also connected to variable threshold comparators to serve as trigger sources. If, as a consequence of an incipient quench, one threshold is exceeded, a trigger is sent to complete the recording process in the ring buffer as well as to initiate actions for magnet protection. As a result the final record is restricted to a window of some hundreds of $\mathrm{ms}$ around the time of quench detection. The acquisition system allows setting the pre-trigger and the post-trigger time intervals as well as the related sampling frequencies.

Besides training curves, a detailed spatial localization of quenches can be pursued by combining information from a voltage taps set and a quench antenna. Voltage taps are implemented in all critical parts of the magnet windings and in the main current leads. During quenches the normal conducting zone propagates in the coils causing the tap potentials to rise. Both direct and differential signals are exploited. The quench antenna, which was described in detail elsewhere [11], collects voltages induced in pick up coils in the magnet bore during the transients provoked by quenches. This is particularly useful for the longitudinal quench localization. Apart from standard power tests, this system proved effective for studies on quench propagation in LHC busbars [12] and for investigations of quench mechanisms and mechanical activity in the quenching magnets [13].

A redundant quench detection system is used as a trigger source to protect the LHC short dipole models during quenches. Each trigger initiates three independent actions: interruption of current supply, dumping of the stored energy in an external resistor and activation of quench heaters. In order to limit the peak temperature in the quenching coils, 


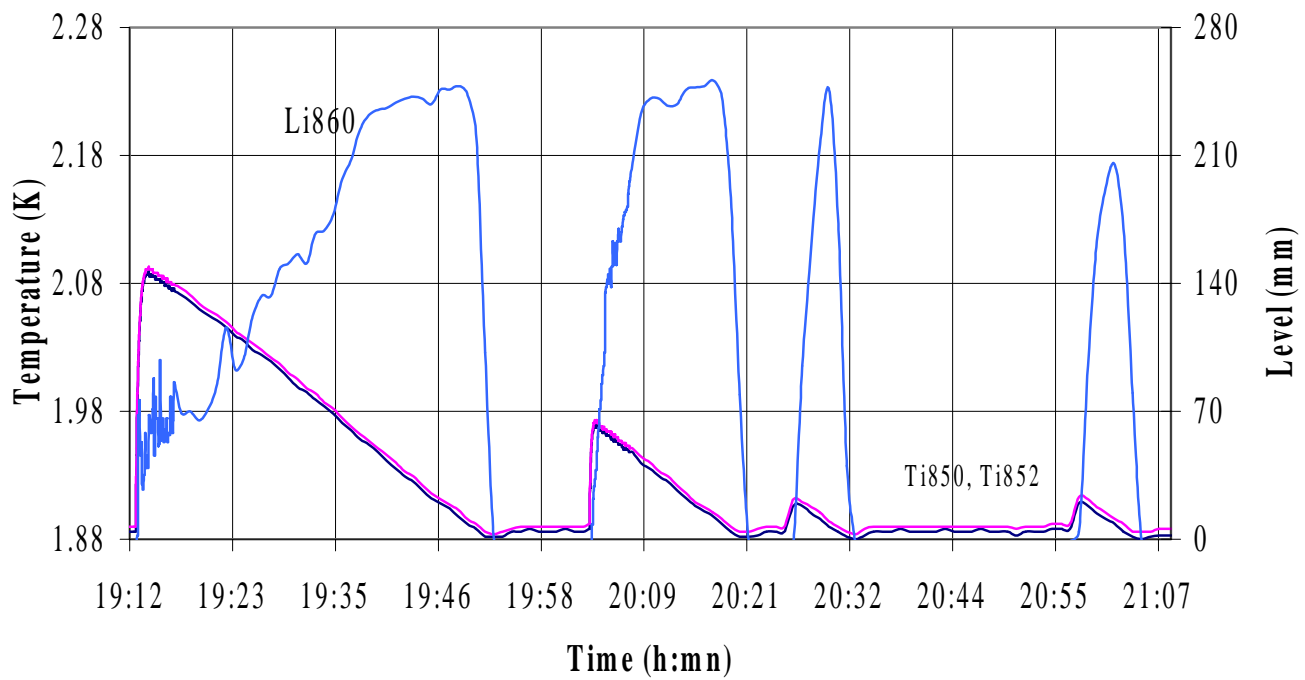

FIGURE 5. Lower bath temperature (Ti850, Ti852) and liquid level in the LBHE (Li860) for three successive quenches at different energies.

fast spreading of the normal conducting zone is provoked by the discharge of capacitors through quench heater strips. From the cryogenics standpoint, quenches cause sudden releases of heat in the lower bath, which must be managed by the control system: the lower bath temperature rises and eventually overcomes the lambda point. The resulting density drop provokes a significant increase of level in the upper bath, and possibly overpressure in the cryostat. Despite temperature excursions in the lower bath, the control system manages to avoid over flooding of the LBHE, as shown in FIG 5.

The power performance of LHC short model dipoles and quadrupoles is studied at 1.9 $\mathrm{K}$, the operating temperature of most LHC magnets. Magnets are also tested at $4.3 \mathrm{~K}$, to check the conductor integrity.

\section{FIELD QUALITY MEASUREMENTS}

The second part of a normal cold test is the measurement of the magnetic field quality [14]. Magnetic measurements targets can be very stringent. For the LHC dipoles an accuracy of $10^{-6}$ relative to the main field component is required on the multipoles. The accuracy goal for field harmonics imposes temperature stability within $10 \mathrm{mK}$, as a consequence of the dependence of filament magnetization on temperature.

Magnetic measurements are performed principally by the rotating coil technique. Calibrated pick-up coils are mounted on glass-fibre vertical shafts, turning immersed in liquid helium in the magnet's bore (FIG 6). The magnet to lambda-plate and cold-warm transitions are equipped with bellows, stiff with respect to torsion but allowing axial misalignments. A DC motor mounted on the top flange of the cryostat drives the shaft via a gear transmission. An angular encoder is mounted on the shaft top. The coil, rotating in the magnetic field, produces a voltage signal, which is integrated by voltage to frequency converters and counters (integrators). The encoder triggers the integrators to start and to reset at equally spaced angular positions. A Labview ${ }^{\circledR}$ based software running on a SUN ${ }^{\circledR}$ Ultra-2 workstation, controls the power supply, the precision current reading, the DC motor 


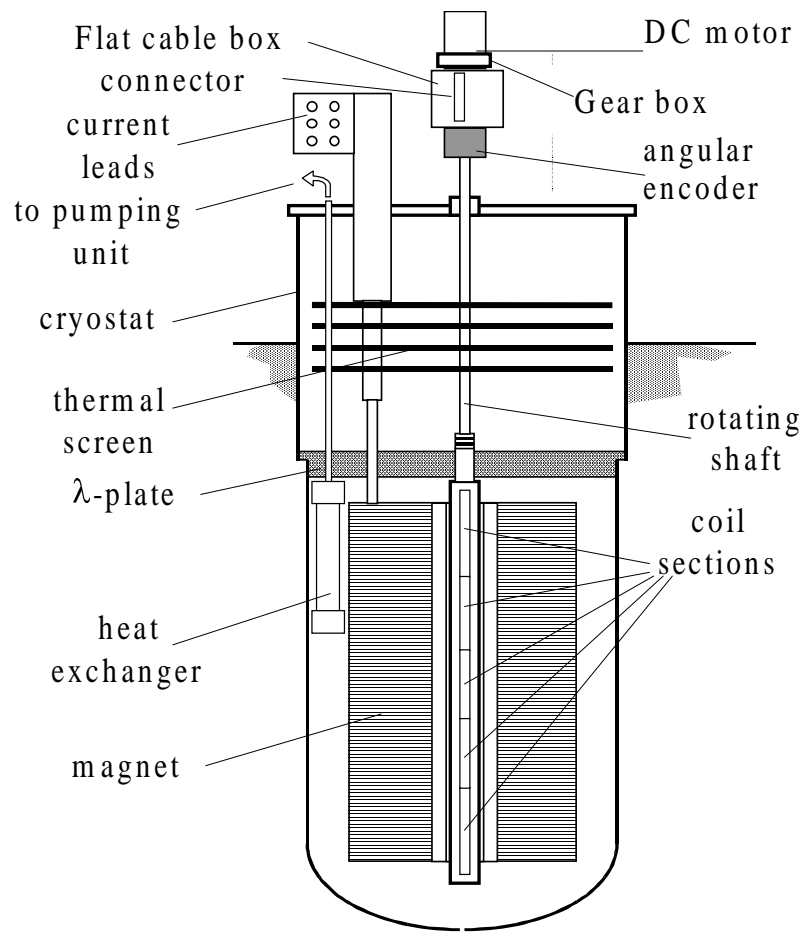

FIGURE 6. Schematic view of the vertical test set-up for a single aperture model magnet.

and the integrators. The raw product of the measurement is a set of magnetic flux increments together with the related angles. The program carries out a discrete Fourier transform to determine the spectrum, which is truncated at a sufficiently high harmonic. Geometric parameters of individual coils, necessary to convert fluxes to magnetic field values, are stored in a separate database. Time resolution, in particular at high fields, is limited by the maximum allowed rotation speed imposed by the saturation of integrators. At a dipole field of $8.3 \mathrm{~T}$ one measurement takes about $25 \mathrm{~s}$ to allow shaft rotation and data transfer through the acquisition hardware.

\section{RECENT AND NEXT UPGRADES}

Besides the short model program, several types of LHC correctors are routinely tested mainly in the Station 3 cryostat. A modular insert was developed allowing testing batches of three different corrector magnets in a single cool down.

New rotation units have been developed to improve uniformity of the rotation speed in magnetic measurements. The former belt transmission has been replaced by gear couplings. A spare insert for Station 1 will be equipped with two pairs of $1.2 \mathrm{kA}$ current leads to allow tests of inner triplet dipole correctors (MCBX) as from October 2001.

With the advent of the LHC series production, the short dipole program, which constituted the principal load for the test facility, is coming to its natural end. A main upgrade of the test station is planned to allow test of LHC pre-series quadrupole magnets for the lattice and the Dispersion Suppressors Short Straight Sections. The cryostat of Station 2 will be removed and replaced with a new cryostat of $5.3 \mathrm{~m}$ length. Civil engineering work is scheduled to start in September 2001, and the first tests are foreseen for the end of the year. 


\section{CONCLUSIONS}

The test facility for the LHC short model dipoles has grown into multitask laboratory for testing several types of superconducting magnets and related equipment. Power performance and field quality measurements are routinely performed. While the short model program is coming to an end, the reception tests of the first series corrector magnets are now taking place. About $10 \%$ of the corrector magnets coming from industries will be cold tested at the Block 4 laboratory before integration in the cold masses of the LHC lattice magnets. The three main cryostats of different sizes will give flexibility to test magnets with overall geometry up to $3.6 \mathrm{~m}$ in length and $0.54 \mathrm{~m}$ in external diameter. The test station has an average capacity of one test set up per week. The "SM18" magnet test plant presently under construction at CERN for the reception tests of all the LHC series magnet assemblies relies on concepts, test procedures and experience gained at Block 4.

\section{ACKNOWLEDGEMENTS}

We owe thanks to B. Galmant, P. Legrand, and to all the present and former members of the Block 4 team.

\section{REFERENCES}

1. Leroy, D., Perin, R., "Development of High-Field Superconducting Magnets for the Large Hadron Collider", in Proc. of the 2nd EPAC, ed. by P Marin and P Mandrillon Ed. Frontières, Gif-sur-Yvette, France, 1990, pp. 1326-1328.

2. Haug, F., Hagedorn, D., Oberli, L., "Cryogenics of the 1.8 K Test Station for 10 Tesla Superconducting Magnet Models" in Proc. of the $12^{\text {th }}$ ICEC, ed. by R.G. Scurlock and C.A. Bailey, Butterworths, London, 1988, pp. 804-809.

3. Billan, J., Gregory, C. R., Legrand, P., Oberli, L., Verveij, A. P., Walckiers, L., IEEE Trans. on Mag. 28, pp. 362-365, 1992.

4. Barth, K., et al., "New Cryogenic Facilities for testing Superconducting Equipments for the CERN Large Hadron Collider" in Proc. of the 17th ICEC, ed. by Dew-Hughes et al., Bristol, 1998, pp. 623-626.

5. Dauvergne, J-P., et al., "A Helium freeze-out Cleaner operating at Atmospheric Pressure" Cryogenics : 34 - pp. 135-138 (1994).

6. Dahlerup-Petersen, K., et al., CERN, LHC Project Report 283, 1999.

7. Augueres, J. L., Aymar, R., Bon Mardion, G., Claudet, G., Faure Brac Plancoulaine, G. J., Senet, L, et al., Cryogenics 20, 9 pp. 529-33, (1980).

8. Giloux, C., "Conception de l'Asservissement du Système de Refroidissement des Aimants Prototypes LHC de 4.3 K à 1.9 K" Conservatoire National des Arts et Métiers, Lyon, France, 2001.

9. Wilson, M. N., Superconducting Magnets, I, 5, p. 68.

10. den Ouden, A., Ten Kate, H., Siemko, A., Sievers, P., and Walckiers, L., "Quench Characteristics of the 11 T Nb3Sn Model Dipole Magnet MSUT" in Proc. of the $15^{\text {th }}$ Int. Conf. on Magnet Technology, ed. by Lin Liangzhen et al., Science press, Beijing, 1997, pp. 339-342.

11. Leroy, D., Krzywinski, J., Remondino, V., Walckiers, L., and Wolf, R., "Quench Observation in LHC Superconducting one meter long Dipole Models by Field Perturbation Measurements", IEEE Trans. Appl. Sup., 3, pp. 781-784, 1993.

12. Herzog, R., et al., "Quench Propagation and Heating in the Superconducting 600 A Auxiliary Busbars of the LHC", and "Quench Propagation in the Superconducting 6 kA Flexible Busbars of the LHC", this conference.

13. Dezillie, B., et al., "Study of Mechanical Disturbances in Superconducting Magnets using Piezoelectric Sensors \& Quench Antenna" Proc. of Int. Symp. of Young Scholars on Mechanics and Mat. Eng. for Science and Experiments, ed by Yichun Zhou et al., Science Press New York Ltd., 2001, pp. 168-170.

14. Jain, A. K., "Basic Theory of Magnets" in Proc. of CERN Accelerator School (CAS), ed. by S. Turner, Geneva, 1998, pp. 1-26. 